Culture et histoire dans l'espace roman

$6 \mid 2011$

Figures du pouvoir dans la littérature hispanoaméricaine

\title{
El teatro peruano, testigo de su tiempo : frente a golpes militares, golpes de voz
}

\section{Elena Guichot Muñoz}

\section{(2) OpenEdition}

\section{Journals}

Edición electrónica

URL: https://journals.openedition.org/cher/9497

DOI: $10.4000 /$ cher.9497

ISSN: 2803-5992

\section{Editor}

Presses universitaires de Strasbourg

\section{Edición impresa}

Fecha de publicación: 30 junio 2011

Paginación: 203-213

ISBN: 978-2-35410-033-9

ISSN: 1968-035X

\section{Referencia electrónica}

Elena Guichot Muñoz, «El teatro peruano, testigo de su tiempo : frente a golpes militares, golpes de VOZ», reCHERches [En línea], 6| 2011, Publicado el 17 diciembre 2021, consultado el 24 enero 2022. URL: http://journals.openedition.org/cher/9497 ; DOI: https://doi.org/10.4000/cher.9497

\section{(c) (i) (2)(2)}

Ce(tte) œuvre est mise à disposition selon les termes de la Licence Creative Commons Attribution -

Pas d'Utilisation Commerciale - Partage dans les Mêmes Conditions 4.0 International. 


\title{
El teatro peruano, testigo de su tiempo: frente a golpes militares, golpes de voz
}

\author{
Elena GUICHOT MuÑOZ
}

Universidad de Sevilla

\begin{abstract}
$\mathrm{M}$
ircea Eliade en su libro El mito del eterno retorno (Eliade 1992: 136)

formula una cuestión esencial para comprender el comportamiento del ser humano frente a la vida: ¿Cómo soportaban los hombres la historia?

En la historia incaica existía una especie de teatro que consistía en que cada Inca mandaba «cantores profesionalizados a componer la historia de sus ancestros, a fin de exaltar los méritos y hazañas de su grupo familiar y de los gobernantes anteriores vinculados a él» (Mazzotti 1996: 31). Estos cantares sostenían una historia oficial ciertamente selectiva pues solo se nombraban aquellos Incas cuyo pasado era favorable recordar, el resto se debía omitir: «el silencio y la omisión era la forma de cambiar el curso de la "historia" [...]. El olvido se apoderaba de los acontecimientos y de las personas» (Rostworoski 2005: 103).

El teatro en esa época era cultivado «en todos los confines del Tahuantinsuyo, $[\ldots]$ las obras se escenificaban los días de fiesta, en lugares públicos y siempre bajo el sol»(Balta 2001: 30). Su función artística, acompañada de la mencionada político-social, no difiere demasiado de la que nos incumbe en el presente estudio, que abarca los últimos veinte años de un grupo que adopta un nombre quechua muy connotativo: Yuyachkani, «estoy pensando, estoy recordando».

Vadearemos los terrenos del mito y de la historia, de la ficción y la realidad, hasta confundirlas en un todo coherente que es la conformación de este excepcional grupo que nace en los años 70 trabajando en sentido
\end{abstract}


opuesto al olvido. Arraigado desde sus inicios en el compromiso ético, su único fin era el de alzar la voz colectiva silenciada durante un período de violencia estancada, que se remonta a la Conquista ${ }^{1}$. En esta década, el teatro sale a las calles, a las plazas, a las universidades para dar lugar a discusiones de corte ideológico, otras veces de estética, que poseen un vértice común: «la voluntad contestataria en política y el seguimiento de las pautas del teatro épico de Bertolt Brecht» (Balta, 2001: 234).

Esta manifestación a nivel nacional no es más que un testigo de su tiempo, una expresión de los cambios sociales que se enmarcan en el Perú del brazo autoritario del General Velasco Alvarado (1968-1975) y de su gobierno militar, que acabó con las esperanzas de todo aquel que anhelara sinceramente una verdadera transformación en este país. Las promesas de la reforma agraria, el modelo de comunidad industrial (Contreras 2007: 332), las sucesivas estatizaciones, quedaron en una pátina superficial, con un cimiento oculto de contrasentidos cuyo saldo final dejó un balance desolador.

Beláunde Terry, en 1980, recoge los destrozos de esa época, que dan lugar, entre otras consecuencias, a una deuda externa desorbitada y a un paulatino crecimiento de la capital, descompensado con la falta de servicios sociales que asistían a la población. No obstante, como recuerda Carlos Iván Degregori ${ }^{2}$ y otros historiadores como Contreras y Cueto, no hay que desestimar el desarrollo del sentido social a nivel nacional que surgió entre los años 70-80, que:

propició, tal vez sin proponérselo, la movilización social, desarrollando en tal sentido una suerte de "revolución cultural" que cuestionó el racismo y el estigma sobre el cual estaban basadas muchas relaciones interpersonales, y en cierta medida homogeneizó las relaciones sociales en el país (Contreras 2007:349)

En el campo dramático es visible el nacimiento de un nuevo teatro popular que pasa por un período de movilizaciones y protestas que

1 Miguel Rubio Zapata, director de Yuyachkani, afirma que «cada vez es menos descabellado pensar que el origen de esta violencia reside en el carácter de imposición que tiene en su origen este Estado, cuyas raíces remontan a la conquista, desde donde arrastramos problemas que aún la sociedad no resuelve» (Rubio 2001: 60).

2 «Esta aparente inmovilidad contrastaba con lo ocurrido en todo el largo periodo "del 63 Belaunde al 80". El Perú había pasado por un conjunto de reformas económicas, políticas, sociales que había provocado redefiniciones de actores y clases y un mayor protagonismo popular en cuanto al reconocimiento de derechos y capacidad de intervención e iniciativa, tanto en los escenarios rurales como en los urbanos» (Degregori 1991: 26). 
desembocan en un «reordenamiento de las fuerzas sociales que se deja sentir en el teatro, remarcando con una práctica nueva su valor crítico y cuestionador del sistema social imperante» (Rubio 2001: 29), representado entre otros grupos por el Teatro Nacional de Perú, de Alonso Alegría. Este nuevo teatro popular apuesta por:

- Una dramaturgia colectiva.

- Nuevos códigos y renovación del lenguaje escénico (con el consecuente cuestionamiento de la autoridad del autor, hasta ahora única voz imperante).

- La noción de grupo.

- Un actor múltiple.

- La mayor conciencia de nuestro papel social

- Un teatro dentro de la cultura nacional (Rubio 2001:32).

Miguel Rubio Zapata, «el ojo de afuera» tal como él se define, director de Yuyachkani, describe el cambio genérico que está germinándose en esta época: «El teatro no es literatura, su fin no es la lectura de textos impresos, sino más bien la lectura escénica ... entendida como la relación espaciotemporal que se da entre la escena y el público» (Rubio 2001: 51).

El grupo nace de la escisión parcial del conjunto teatral llamado Yego, teatro comprometido. Su primera obra, Puño de Cobre, de 1971, se encuadra más en la corriente del Teatro de Arena Sao Paulo de Augusto Boal, dentro del proceso de nacionalización del escenario. Se trata de un acto teatral de oposición a la explotación de mineros que ejercía la Cerro de Pasco Corporation, empresa extranjera que dejó como saldo la muerte de veinticinco mineros en la población de Cobriza, tras una legítima marcha de los trabajadores en protesta por sus condiciones. Teresa Ralli, una de las actrices del grupo, acude a esta población sensibilizada por el tema y descubre la siguiente reacción del público tras la representación de la pieza:

La presentamos ante los trabajadores - nosotros habíamos ido en blue jean, cafarena, nada más; sin maquillaje ni nada - y después que terminamos, ellos nos mostraron una danza hermosa, como agradecimiento; y nos dijeron que qué pena que nos hubiéramos olvidado nuestros disfraces, que seguro para la próxima ya los íbamos a llevar... Nosotros no nos habíamos olvidado de nada; y cuando nos mostraron su danza, empezamos a observar cómo era nuestra cultura, cómo era nuestro país; donde cada color, cada vestido, cada música, es un código de comunicación con el otro, con el que estás dialogando. ¡Ahí se nos abrió el mundo! (Ralli 2009).

Infiere de esta experiencia la necesidad de imbuirse en la complejidad cultural que querían representar y la imperiosa capacidad de la fuerza de la oralidad originada del diálogo constante con los propios presos de Cobriza. 
Así, Miguel Rubio afirma que «Sólo el pueblo es capaz de ofrecer raíz, identidad y destino a nuestras naciones. Cuando los personajes populares han subido a la escena, lo han hecho reflejando su papel en la forja de su destino. Es decir, ya no como comparsa sino como protagonistas de su historia» (Rubio 2001: 35). En constante cambio y ebullición, Yuyachkani va desmarcándose de sus inicios, provocando una discusión teórica que acaba concibiendo un término específico para nombrar a este movimiento latinoamericano: El Nuevo Teatro.

Kati Rögger (Kohut 1998: 238) expone una serie de características que definen este teatro, cuyas bases teóricas descansan tanto en los escritos de Enrique Buenaventura y la fundación del Teatro Experimental de Cali (TEC), el Teatro de la Candelaria de Santiago García, como en la realidad histórica que va demandando una mutación incesante. En primer lugar, tras la experiencia de Cobriza deciden oponerse de forma absoluta a la «instrumentalización política y didáctica del teatro» (Rubio 2001: 239). Continúan trabajando con un teatro político, en el sentido en que su objetivo es exponer «las contradicciones político-sociales imperantes en una realidad acuciante» (Artesi 1989: 83), pero discrepan del hecho de "aceptar que hay que dar un teatro de baja calidad al principio para luego ir elevando al nivel del público... "esta acción" es postular que el público no está maduro para la libertad» (Watson 1978: 239).

La segunda es que este Nuevo Teatro nace de una relación abierta con el público que implica un papel activo del espectador, por lo que exige ir más allá de lo que propugnaba «El teatro del oprimido» de Boal, o «El Teatro Popular» de los 70. La intención de Yuyachkani desde el principio es la de «no subestimar al espectador, sintiéndonos en la obligación de explicarle todo. Mejor dejarle preguntas que intentar darle respuestas». (Rubio 2001: 74). En consecuencia, trabajan por la elaboración de una obra abierta: «lo importante es que la imagen que proyecta la escena sea sugerente, abierta y parcial y que además permita al espectador hacer lo suyo, es decir, completar la imagen y elegir su propio orden o desorden, si así lo prefiere» (Rubio 2001: 190).

Para ello, se sirve de una tercera característica que recoge una de las grandes corrientes del teatro latinoamericano « un concepto de dramaturgia abierta que no se basa en el teatro como género literario, sino en la práctica del montaje y en la invención creadora del actor: la creación colectiva", sinónimo, según Kati Röger, de «teatro de la incertidumbre», pues "produce intencionalmente una crisis para superarla después» (Kohut 1998: 241). Los 
medios de expresión son múltiples y variados: se valen de danzas populares, máscaras, y sobre todo del lenguaje técnico-corporal; nuevas creaciones que ya no quieren ser un espectáculo "tributario del texto literario", sino una apertura a "una noción teatral totalizadora (Pérez 1989: 35).

Por último, se destaca en este Nuevo Teatro la invención de personajes nacionales: "El personaje tiene que ser concebido como un espacio de contradicciones de la sociedad, incluyendo en este espacio las causas de las contradicciones de una época, porque no se limita a las contradicciones de si mismo sino a las contradicciones de los códigos» (García, 1983: 86).

Como podemos observar, este teatro muestra palpablemente una adhesión absoluta al contexto socio-histórico sin caer en la mera referencia realista o naturalista, sino generando una postura estético teatral que pueda dar explicación al mosaico cultural que se plantea como problemática del Perú. En 1985, la Muestra Nacional de Teatro fundada por MOTIN (Movimiento de Teatro Independiente) se erige como un hito en la consolidación de este Nuevo Teatro. Yuyachkani comienza a germinar un giro que parte de la reescritura del mito enmarcada en un período de la Historia del Perú reinado por la corrupción institucionalizada.

Para entender este proceso de transformación es necesario ofrecer paralelamente ciertos parámetros históricos que lo iluminen. Desde mediados de los 80 se generaliza en el escenario teatral peruano un intento de crear una "restitución simbólica» (Salazar 1990: 18) sobre la identidad peruana que se enfrente con las formas del poder omnímodo que corroen la escena nacional. Estos actos teatrales responden de forma intuitiva a lo que Hugo de Salazar denomina "una cultura de la violencia» $»^{3}:$ "El asqueroso maquillaje de la guerra sucia, la masacre, la desaparición forzosa, la tensión, el pánico contenidos, pasan a ser alegorías, metáforas, mensajes en clave ritual que como un susurro entre dientes empiezan a invadir desde las butacas la escena teatral peruana» (Salazar 1990: 9-10).

Las tres obras que trabajamos dentro de este estudio se enmarcan en el clímax de esta «cultura de la violencia» que actúa de forma silenciada durante más de veinte años. Nos situamos en la segunda reelección del gobierno del Presidente Alberto Fujimori definido por su naturaleza castrense y autoritaria. Tras el autogolpe que promulgó en 1992, el control del

3 Hugo Salazar del Alcázar extrae el término «cultura de la violencia» del artículo: "Violencia y pacificación», Comisión especial del senado sobre las causas de violencia y alternativas en el Perú, Desco, Comisión Andina de Juristas, Lima, 1989. 
poder Ejecutivo abarcó todas las funciones. El SIN, dirigido por Vladimiro Montesinos, se encargó de la vigilancia y castigo de todo acto subversivo, hasta ampliar su poder sobre el propio líder del gobierno, Alberto Fujimori, instaurándose un régimen de hostigamiento a los opositores con el manejo de los medios, un sistema enmascarado bajo una fachada democrática que impedía la intervención de fuerzas externas (véase Cotler 2001: 53).

Incrementando aún más este clima de manipulación tiránica de la realidad, se erigieron dos frentes que supusieron una sistemática violación de los derechos humanos, tal como pone de manifiesto el equipo de la Comisión de Verdad y Reconciliación en su Informe final sobre esta «guerra sucia» encubierta. Por un lado, nos encontramos con la presencia de las organizaciones subversivas - el Partido Comunista del Perú Sendero Luminoso y el Movimiento Revolucionario Túpac Amaru, este último «en una escala notoriamente más pequeña» (Lernes 2004: 191):

El Partido Comunista del Perú, conocido como Sendero Luminoso (PCPSL), desencadenó en 1980 el más violento conflicto armado contra el Estado y la sociedad peruana de la historia de la República, a lo largo del cual, según constató la CVR, se cometieron gravísimos crímenes que constituyen delitos de lesa humanidad, siendo el responsable del 54\% de las muertes y desapariciones reportadas a la CVR [...] la cifra total de víctimas totales ocasionadas por dicha organización subversiva asciende a 31.331 personas (Lernes 2003: 13).

Por otro, el propio gobierno consintió a los cuerpos policiales y a las fuerzas armadas actuar impunemente bajo el pretexto de la defensa nacional:

la autoridad civil desatendió durante mucho tiempo las miles de denuncias de violaciones de los derechos humanos cometidas por las fuerzas del orden en las zonas más afectadas por el conflicto... los agentes del Estado - Fuerzas Aramadas y Policía -, los comités de autodefensa y los grupos paramilitares son responsables del $37 \%$ de los muertos y desaparecidos reportados a la CVR (Lernes 2004: 18-19).

Hatun Willakuy, vocablo quechua que significa «El Gran Relato», es el título del Informe que la Comisión de la Verdad y la Reconciliación hizo público en el año 2003, cuya misión es la de corregir la Historia que fue falseada y manipulada durante esta época de terror. Antonio Cornejo Polar nos explica la inefabilidad, la ausencia de opción en el lenguaje que describe los horrores de la realidad peruana, que desde Arguedas, enmudece a los escritores por "la violencia sin límites que desgrana al país» (Kohut 1998: 25). No es este el caso del grupo Yuyachkani, que ante la violencia 
asestada, irrumpe con más fuerza, y vuelca toda la imaginación a la escena para mostrar esta "realidad de muerte» (Rubio 2001: 104) en la que los más afectados siguen siendo los miembros de la población campesina, los más desposeídos. Miguel Rubio Zapata afirma que precisamente en este momento «no podemos hacer obras en la[s] que se utilice el ellos como algo lejano y distinto » (105).

El ritual y el mito se integran en el discurso estético para descartar reducciones maniqueas que impidan profundizar en todo el horror acontecido en estos años. Aunque reivindiquen y no disimulen el lado social insisten en que crear «una obra de teatro en registro mítico» parte de "reivindicar el derecho al sueño, a la invención». No se trata pues de «representar mitos» porque «el hecho mismo de llevar un mito al teatro, por más exacto que sea, está siendo desvirtuado por estar en un contexto ajeno a su origen y su función", sino de reflejar "el mito en tanto a sueño [...] como simbolización que partió del límite del ser humano al no tener explicaciones "coherentes" de su realidad» (98-99).

Mircea Eliade da una explicación antropológica a este tipo de manifestaciones artísticas: «La formulación en términos modernos de un mito arcaico delata, por lo menos, el deseo de hallar un sentido y una justificación transhistórica a los acontecimientos históricos» (Eliade 1992: 141). La instauración de estas historias vertebradas por distintos mitos busca ante todo «irrumpir en el cotidiano de los pobladores, y sorprenderlos en un diálogo con la teatralidad a través de la fábula, la danza, la imagen y la música para de esta manera remover la memoria y generar una nueva mirada sobre la historia vivida en los últimos veinte años» (Diéguez 2009: 111).

Tres obras definen este acto de visibilidad, de resistencia contra el olvido: Adiós Ayacucho (1990), Antígona (2000) y Rosa cuchillo (2004). Adiós Ayacucho representa la historia de Alfonso Cánepa, dirigente campesino torturado y mutilado por las fuerzas armadas, que queda enterrado en una fosa común. Un Q'Olla de Pautarcambo, figura cómica «de una comparsa de danzantes cusqueños» (Rubio 2006: 147), asume su cuerpo y decide ir de Ayacucho a Lima para pedirle al Presidente los huesos que no pudo sepultar: «El cadáver es, como si dijéramos, la unidad mínima de la muerte, y dividirlo como se hace hoy en el Perú es quebrar la ley natural y la ley social» (Ortega 2008: 43).

La primera asociación mítica que establecemos nos conduce al Inkarri, mito según el cual el cuerpo descuartizado del Inca ha de recomponerse de la tierra para renacer; pero se trata de un «Inkarri contemporáneo » que 
mermado de paciencia, "decide ir en busca de los huesos que le faltan» (Rubio 2001 :92). Asimismo esta descomposición del cuerpo en pedazos nos conduce inequívocamente a la imagen homérica de Héctor, que tras perecer en una encarnizada lucha a manos de Aquiles, es arrastrado en un carro de combate, hasta acabar con el cadáver. El objetivo del vencedor parte de un deseo de humillación y de corrupción integral del enemigo que altera profundamente «los códigos de honor de la propia épica» (Camacho 2010). El crítico José Manuel Camacho explica cómo Aquiles desobedeció las leyes de la guerra antigua según las cuales era imperativo ofrecer el cuerpo del derrotado a sus adversarios para que pudieran velarlo. Así, nos alumbra sobre una trágica particularidad de la historia peruana: «la confirmación de que la historia trágica del hombre parece dar vueltas ad infinitud sobre el eje de la crueldad y se alimenta del más imperecedero de los sentimientos: el odio» (Camacho 2010).

Antígona recupera el mito de Sófocles para hablarnos de la perversión del poder mostrando una historia muy similar a la de muchas madres y hermanas que no pudieron enterrar el cuerpo de sus familiares desaparecidos: «Murió por un juego perverso de los dioses. [...] Ellos observan las batallas como un espectáculo, ignorando quién hiere a quién en el fragor del combate o que flecha lleva dirección de cuerpo preciso» (Watanabe 2008: 246).

Rosa Cuchillo es la historia de una mujer ancashina que busca más allá de la muerte a su hijo desaparecido, deambulando por los tres mundos que rescatan la cosmovisión andina: el Kay Pacha (nuestro mundo), el Uqhu Pacha (el mundo de abajo), y el Janaq Pacha (el mundo de arriba) ${ }^{4}$.

Los componentes del grupo Yuyachkani se implican en la acción directa dentro de la campaña iniciada por la CVR titulada "Para que no vuelva a suceder» (Cox 2004: 206), acudiendo a ciudades andinas donde eran convocadas Audiencias Públicas que daban testimonio de las atrocidades cometidas. En un proceso de continua innovación deciden borrar las fronteras entre el teatro y la vida, y comienzan a incluir testimonios verídicos en la conformación de estas piezas, creando un mosaico de voces, para que «la memoria no se borre y la condición humanista no sea un tema de

4 Esta representación es la que más difiere de su texto original. Podríamos decir que el texto de Óscar Lucio Colchado, del que nace Rosa Cuchillo, es una semilla de la producción de Yuyachkani. Ana Correa, única portadora de la acción escénica, explica las fuentes de su creación e investigación: Ana Correa «Sanaciones y reparaciones simbólicas», Karpa 2.1., Dissident Theatricalities, visual arts and culture. Disponible en internet: http://www. calstatela.edu/misc/karpa/Karpa2.1/Site\%20Folder/Resources/rosacuchilloanacorrea.pdf 
los discursos literarios» (Rubio 2006: 19). Rosa Cuchillo se alimenta de un personaje real llamado Angélica Mendoza, Mamá Angélica, madre de Arquímedes Ascarsa Mendoza, secuestrado y desaparecido en Ayacucho, el 12 de Julio de 1983. Parte del texto que representa Ana Correa son testimonios de esta madre-coraje.

En el curso de la preparación de Antígona, Teresa Ralli convocó a un grupo de mujeres madres y hermanas de desaparecidos que le permiten «entrar en diálogo con su realidad cotidiana de lucha por encontrar los cuerpos de sus maridos y hermanos para darles sepultura ", y a raíz de este encuentro logra encontrar la presencia física que está buscando.

Todas estas piezas tienen precisamente su origen en textos literarios de diferentes autores peruanos; respectivamente, de Julio Ortega (Adiós Ayacucho, 1984), de la versión libre de Antígona de José Watanabe (2000), y de la obra homónima de Óscar Lucio Colchado (1997). Sin embargo, el soporte textual será deliberadamente modificado, amputado, con la intención de fundir la palabra con los múltiples códigos alternativos que suponen una parte esencial del mundo andino - excepto en el caso de Antígona, que será escrita por el poeta peruano expresamente para el grupo cultural Yuyachkani. El autor se transforma así en parte del proceso teatral, en una cadena horizontal donde no existe una jerarquía predeterminada, con el fin de que «las sensibilidades de la masa transeúnte se conecten en varios niveles con la colectividad teatral» (Rubio 2001).

De forma voluntaria, se está desarticulando una tradición literaria que imponía al autor como entidad discursiva preponderante. En palabras de Cornejo Polar, nos establecemos ahora «entre la voz de las culturas ágrafas andinas y la letra de la institución literaria de origen occidental, con su abigarrada e inestable gama de posiciones intermedias, hasta la transcripción de la palabra hablada en el testimonio y la construcción del efecto de oralidad en el discurso literario" (Cornejo Polar 1994: 11).

De esta necesidad parte precisamente el teatro de creación colectiva, que a través de la multidisciplinariedad de su proyecto artístico dota al público de un atributo fundamental: la presencia viva. Alfonso del Toro, en su libro Estrategias postmodernas, y postcoloniales en el teatro latinoamericano actual, sintetiza las nuevas corrientes teatrales en Latinoamérica en torno a cuatro nombres específicos: postcolonialidad, hibridez, cuerpo y teatralidad (De Toro 2004: 9-20). Es más que evidente la coincidencia de estos términos con aquellos que definen al grupo cultural Yuyachkani. No obstante hay uno que prevalece sobre el resto: la importancia del cuerpo, que el crítico define 
como «la marca para la materialidad, para representaciones mediales de la historia del colonialismo (memoria, inscripción, registro), de la opresión, tortura, manipulación, agresión y confrontación» (16).

Miguel Rubio Zapata escoge la materialidad, la presencia como clave de Yuyachkani:

Presencia podría ser entonces el hilo visible y la palabra clave para señalar el sentido de la técnica en que se han orientado nuestros actores desde el inicio... De la presencia heroica, militante de los primeros años, a la posterior búsqueda de la presencia mediante las acciones físicas y luego a la presencia del estar con y sin acciones que trabajamos ahora; siempre presencia, sin ella no existimos y estamos doblemente ausentes en el escenario (Rubio 2001: 162).

Frente al afán de las figuras de poder por usurpar toda identidad, toda entidad, se impone una presencia siempre en guardia que nos ayuda a recomponer los «escenarios liminales $»^{5}$, la invisible frontera que existe entre el teatro y la vida, entre la ausencia y la presencia, entre el actor, y el ser humano:

No sólo la realidad y la ficción parecieron eliminar fronteras durante los procesos creativos y las funciones de estas obras. Algunas veces, durante las audiencias públicas, pobladores humildes de origen campesino se acercaron a los personajes a ofrecer sus testimonios. En Vilcashuamán los campesinos salieron despavoridos cuando se encendieron los pequeños cuetes que se usan en Adiós Ayacucho. Todo se ha mezclado, todo se ha removido al agitarse la memoria (Rubio 2006: 63).

\section{Bibliografía}

Artesi, Catalina Julia, "Algunas modalidades del teatro político hispanoamericano ", 1989, en Instituto Internacional de Teoría y Crítica de Teatro Latinoamericano, Reflexiones sobre teatro latinoamericano del siglo veinte, Buenos Aires, Galerna, 1989, pp. 83-93.

Balta Campbell, Aída, 2001, Historia general del teatro en el Perú, Lima, Universidad San Martín de Porres.

5 Véase con atención el siguiente artículo de Ileana Diéguez, 2006, «Escenarios liminales: donde se cruzan el arte y la vida (Yuyachkani, más allá del teatro...)», Artea, Investigación $y$ arte escénica. Disponible en internet: http://artesescenicas.uclm.es/archivos_subidos/ textos/41/EscenariosLiminales.pdf. La autora hace hincapié sobre la idea de lo liminal desde las visiones de Victor Turner y Rocco Mangieri en relación con diversas piezas de Yuyachkani estrenadas desde los 90. 
Camacho Delgado, José Manuel, 2010, «Aquiles en los Andes. El odio y sus máscaras en la narrativa peruana de la violencia», Tinta China, № 12-13, año IX, marzo 2010. Disponible en internet: http://www.tinta-china.net/j_m_camacho_12.htm

Contreras Carlos y Cueto, Marcos, 2007, Historia del Perú contemporáneo, Lima, Instituto de Estudios peruanos.

Cotler, Julio y Grompone, Romeo, 2001, El fugimorismo: ascenso y caída de un régimen autoritario, Lima, Instituto de Estudios Peruanos.

Cornejo Polar, Antonio, 1994, Escribir en el aire: ensayo sobre la heterogeneidad socio-cultural en las literaturas andinas, Lima, Horizonte.

Cox, Mark, 2004, Pachaticray (el mundo al revés), Lima, San Marcos.

De Toro, Alfonso (ed.), 2004, Estrategias postmodernas y postcoloniales en el teatro latinoamericano actual: hibridez, medialidad, cuerpo, Madrid, Iberoamericana.

Degregori, Carlos Iván y Grompone, Romeo, 1991, Elecciones de 1990: Demonios y redentores en el nuevo Perú: una tragedia en dos vueltas, Instituto de estudios peruanos, Lima.

Diéguez, Ileana y Araújo, Raque, 2009, Des/tejiendo escenas: desmontajes: procesos de investigación y creación, México, Documentación e Información Teatral Rodolfo Usigli.

Eliade, Mircea, 1992, El mito del eterno retorno, Madrid, Alianza Editorial.

García, Santiago, 1983, Teoría y práctica del teatro, Bogotá, CEIS.

Kohut Karl, Morales Saravia José., Rose Sonia (eds.), 1998, Literatura peruana hoy: crisis y creación, Frankfurt am Main - Madrid, Vervuert/Iberoamericana.

Mazzotti, Jose, 1996, Coros mestizos del Inca Garcilaso. Resonancias andinas, Lima, Fondo de cultura económica.

Ortega, Julio, 2008, Adiós Ayacucho, Lima, Fondo Editorial de la UNMSM.

Pérez Coterillo, Moisés (dir.), 1989, Escenarios de dos mundos: inventario teatral de Iberoamérica, Vol. I, Madrid, Centro de Documentación Teatral.

Ralli, Rebeca, 2009, «Nosotros queríamos cambiar el mundo», 24 del abril 2009, El comercio. Disponible en internet: http://blogs.elcomercio.pe/ejecutivas/2009/04/ rebeca-ralli-nosotros-queriamo.html

Rostworoski, María, 2005, Estructuras andinas del poder: Ideología religiosa y política, Lima, Instituto de Estudios Peruanos.

Salazar del Alcázar, Hugo, 1990, Teatro y violencia: una aproximación al teatro peruano de los 80, Lima, Jaime Campodónico. Watanabe, José, 2008, Poesía Completa, Madrid, Pretextos.

Watson Espener, Maida, Reyes, Carlos José, 1978, Materiales para una historia del teatro en Colombia. ICC, BBC, 33, Bogotá. 